\title{
REDESCUBRIMIENTO DE UNA ESPECIE DE CALCEOLARIA (CALCEOLARIACEAE)
}

\section{REDISCOVERY OF A CALCEOLARIA SPECIES (CALCEOLARIACEAE)}

\author{
Mélica Muñoz-Schick ${ }^{1}$ \& Andrés Moreira-Muñoz ${ }^{2}$ \\ ${ }^{1}$ Museo Nacional de Historia Natural, Casilla 787, Santiago, Chile \\ ${ }^{2}$ Instituto de Geografía, Pontificia Universidad Católica de Chile
}

\begin{abstract}
The species Calceolaria reicheana, nomen nudum, was rediscovered in the Metropolitan Region, Chile. It is considered as a valid species, described and renamed as Calceolaria espinosae. A new locality is also recorded.
\end{abstract}

La especie Calceolaria reicheana Espinosa (nom. nud.)fue señalada para la región Metropolitana, cumbre del cerro El Roble y cerros de Montenegro, en 1933 por Marcial R. Espinosa, en una sesión ordinaria de la Sociedad Chilena de Historia Natural (Revista Chilena Hist. Nat. 37: 313, 1933, publ. 1934). Este nombre sólo fue leído en dicha ocasión, pero no se realizó una descripción de la planta, por lo tanto es un nomen nudum.

Ehrhart en su monografía del género (2000: 277), también hace mención al nombre, pero la señala como dudosa porque no la encontró en la naturaleza. Al revisar el material de herbario se ha constatado que existen en SGO sólo los ejemplares de Montenegro recolectados por Espinosa, pero no los de cerro El Roble.

En el año 2002 se recolectó en la cumbre del cerro El Roble y se ha seguido observando allí, confirmando que corresponde a una buena especie. Espinosa se la dedicó a Carlos Reiche, pero por las reglas de nomenclatura no se puede usar ese nombre y se la dedicamos a M. Espinosa, primer recolector de la especie.

Calceolaria espinosae M.Muñoz et Moreira nov. sp. TIPO: Chile, Región Metropolitana, Prov. Chacabuco, cumbre cerro El Roble, $32^{\circ} 59^{\prime}$ Lat. S, $71^{\circ} 01^{\prime}$ Long. W, 2.222 msm, A. Moreira 859, 31-XII-2002 (Holotipo SGO 150135) (Fig. 1).

Planta perennis, tote piloso-glandulosa. Caules 12 nodati, folia elliptica, rugosi, tote pilosa, pilis albi-glandulosis, margine entire vel brevi dentatis. Corolla lutea, sparce glandulosa, labiis rotundis, striatis, superiore minor ad inferiorem longae. A species $\mathrm{C}$. meyeniana differt corolla labiis plus latus, superior plus planus, et folia tote pilosa.

Planta perenne, quebradiza, de 12-20 (-25) cm de alto, enteramente piloso-glandulosa. Tallos decumbentes, algo rojizo-amarillentos, leñosos en la base, 1-2nodes, pelos blancos, los cortos densos y los largos de 1,5-2 mm de largo, más espaciados. Hojas principalmente en la base, láminas elípticas, rugosas, de $12-15 \mathrm{~mm}$ de largo por 10-12 $\mathrm{mm}$ de ancho, las basales prolongadas en un pseudopecíolo de 3-5 $\mathrm{mm}$ de largo, enteramente cubiertas de pelos blancos, glandulosos, de 1-3 mm de largo; el margen es liso o con dientes poco notorios. Primer par de hojas tallinas brevemente pecioladas, $2^{\circ}$ par sésil. Inflorescencia cimosa, bifurcada 2 veces, pequeñas brácteas foliosas en la base de las bifurcaciones. Flores con pedicelos finos, cáliz con sépalos ovados, piloso-glandulosos, de 3,5-4 $\mathrm{mm}$ de largo, corola amarillo-oro, sin pintas, algo glandulosa, labios inflados mayores que el cáliz, forma redondeada, pero angostada hacia los lados, el inferior de $1,1 \mathrm{~cm}$ de largo por 1,3 cm de ancho, el superior menor que el inferior, erecto, algo abierto y de forma más aplanada, ambos con estrías que les da un aspecto rugoso-almenado; el labio inferior tiene una parte hundida en la garganta donde se apoyan estambres y pistilo; los estilos son de 3,5 mm de largo, con 
tecas de desarrollo igual entre sí, de 1,5 mm de largo. Cápsula glandulosa, piriforme, de base ancha y angostándose hacia el ápice, de 7-8 mm de largo, rodeada por el cáliz en 3/4 de su largo. Semillas de 0,6 $\mathrm{mm}$ de largo por $0,3 \mathrm{~mm}$ de ancho, café-anaranjadas, con superficie transversalmente surcada (Fig. 2 B-H).

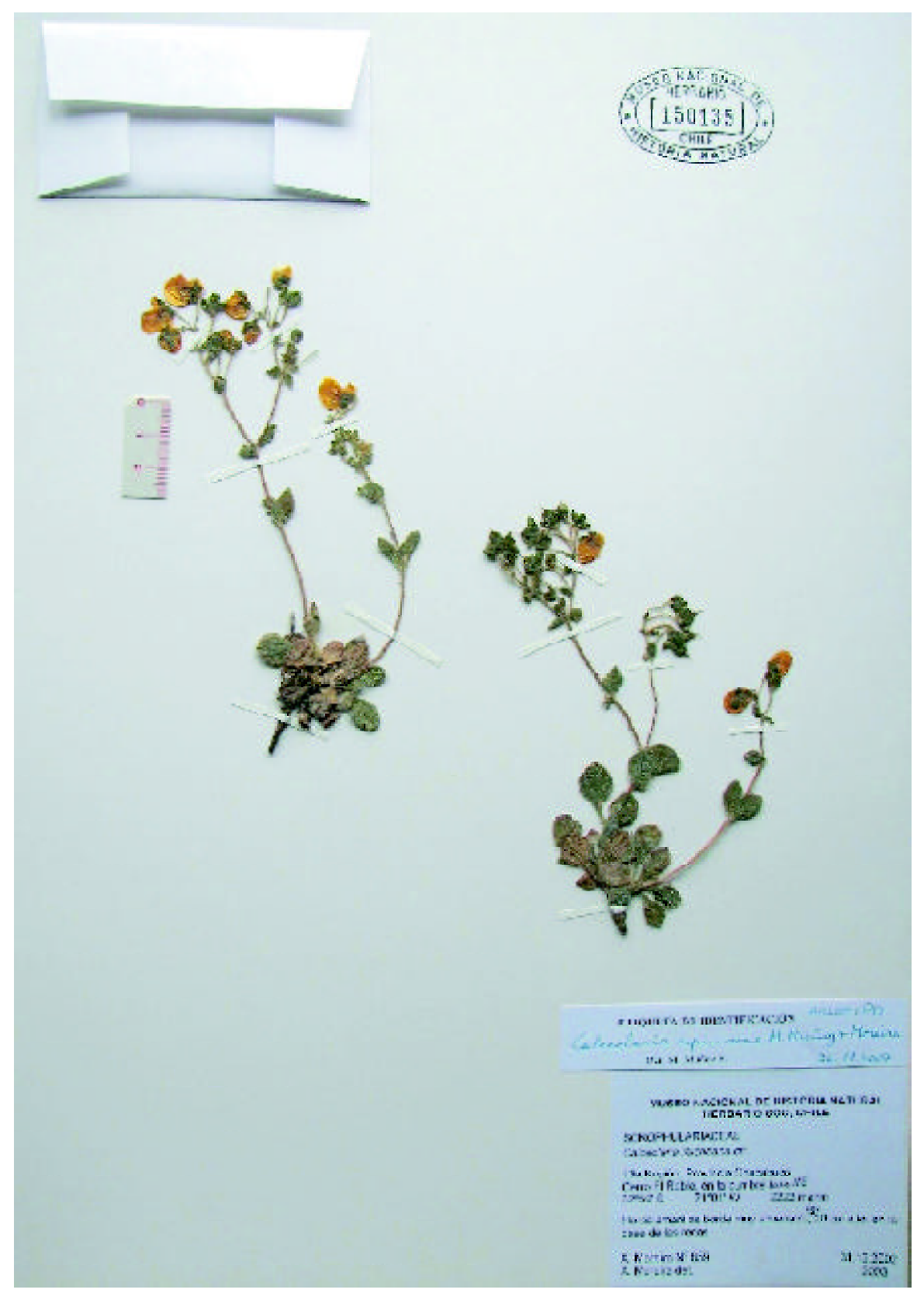

Figura 1. Calceolaria espinosae M.Muñoz et Moreira, HOLOTIPO (Fotografía de V. Ardiles).

Figure 1. Calceolaria espinosae M.Muñoz et Moreira, HOLOTYPE (Photography by V. Ardiles). 


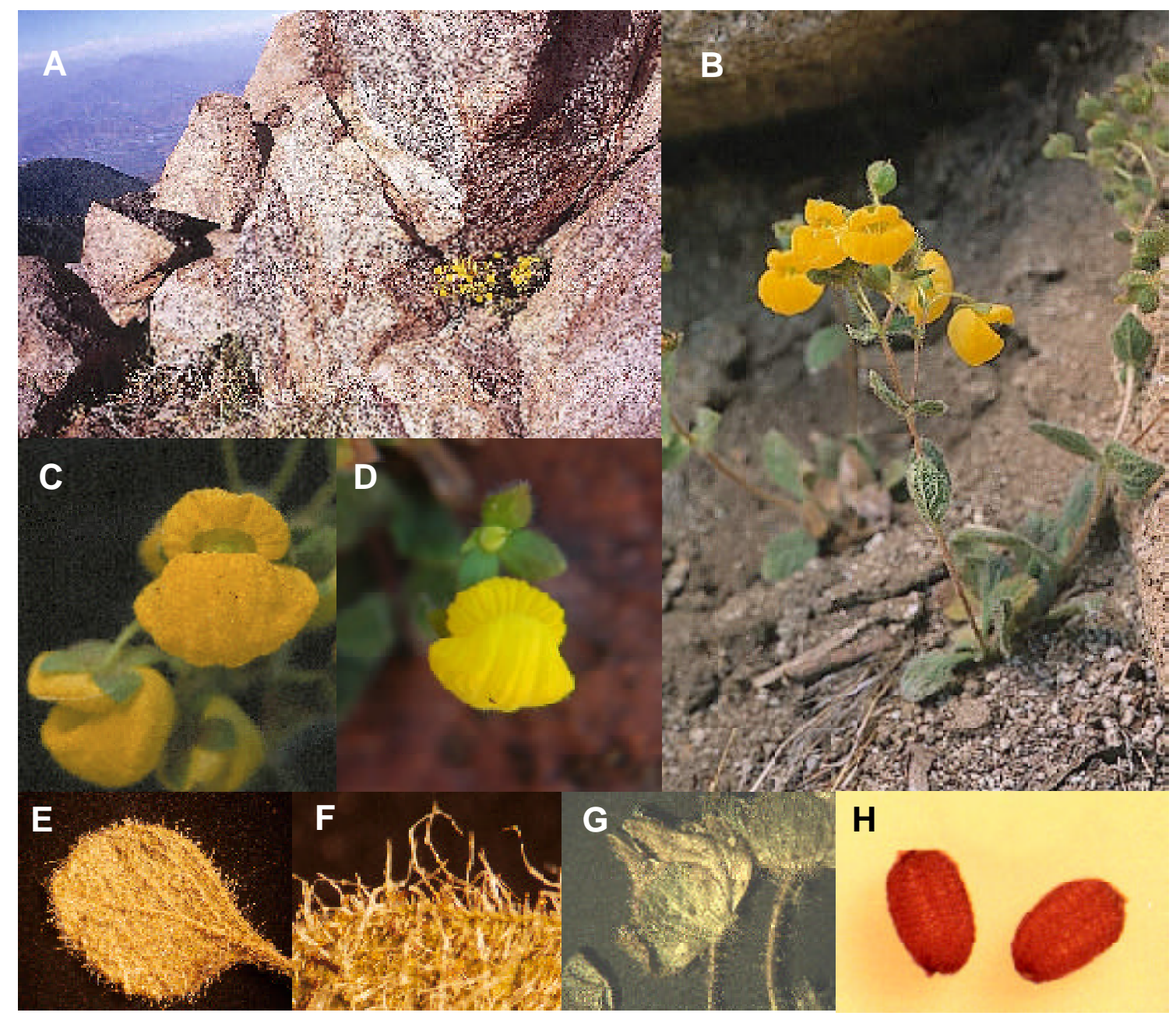

Figura 2. Calceolaria espinosae M.Muñoz et Moreira. A. Hábitat; B. Hábito; C-D. Detalle de flores (x2); E. Hoja envés (x2); F. Detalle de los pelos; G. Cápsula (x3); H. Semillas (x25) (Fotografías: A. A. Moreira; B-C. S. Elórtegui; D. M.T. Eyzaguirre; E, F, H. E. Barrera; G. A. Vidal).

Figure 2. Calceolaria espinosae M.Muñoz et Moreira. A. Habitat; B. Habit; C-D. Flowers (x2); E. Leaf beneath (x2); F. Hairs detail; G. Capsule (x3); H. Seeds (x25) (Photos: A. A. Moreira; B-C. S. Elórtegui; D. M.T. Eyzaguirre; E, F, H.E. Barrera; G. A. Vidal).

MATERIAL ADicionAl:

Región Valparaíso: Antes del portezuelo, al N del pueblo de Pedernales (3202’ 3,5’'S, 7049’ 4,3’W), $1.824 \mathrm{msm}$, 17-XI-2007, P. von Fürstenberg (SGO 155240).

Región Metropolitana: Prov. Melipilla, Montenegro, 5-XI-1933, M.R. Espinosa(SGO 64175, 64176, 64177); Prov. Chacabuco, cumbre cerro El Roble, cima NE,
(3258'5,'’'S, 71 0 0,8' 9' 'W), 2.222 msnm, 965, 27-XI2005, A. Moreira (SGO 154411); Prov. Chacabuco, cumbre cerro El Roble, cima NE, $\left(32^{\circ} 58^{\prime}\right.$ '5,'’' $71^{\circ}$ 0,8'9"W), 25-XI-2007, M. Muñoz 4880 (SGO 155241); Prov. Chacabuco, cumbre cerro El Roble, cima NE, $\left(32^{\circ} 58^{\prime} 5,2\right.$ ' $\mathrm{S}, 71^{\circ} 0,8^{\prime} 9$ ' $\left.\mathrm{W}\right)$, en fruto, 20I-2008, M. Muñoz 4890 (SGO 155242). 
La especie descrita es similar a C. meyeniana Phil., en cuanto a la forma de la flor, aunque en C. espinosae el labio inferior es algo más ensanchado hacia los lados y el labio superior es algo aplanado. Las especies se diferencian por el hábito subarbustivo y tallos erectos en $C$. meyeniana (30-70 $\mathrm{cm}$ alto), en cambio $C$. espinosae es hierba perenne hasta $25 \mathrm{~cm}$ de alto $\mathrm{y}$ tallos decumbentes. Por otra parte, ninguna de las subespecies de $C$. meyeniana presenta pilosidad tan larga y densa como en C. espinosae.

Las plantas de C. espinosae crecen protegidas entre las fisuras de grandes rocas antes del portezuelo al norte de Pedernales, en el límite entre las regiones de Valparaíso y Coquimbo; similar hábitat ocupan entre rocas graníticas en la cima del cerro El Roble, generalmente en exposición nor-este, sólo unas pocas se disponen hacia el poniente (Fig. 2 A). En el año 2007 se observaron alrededor de 15 plantas, la mayoría en plena floración, algunas en fruto.

Otras plantas presentes entre los roqueríos son Maytenus boaria Molina, arbusto achaparrado, Nardophyllum lanatum (Meyen) Cabrera, arbusto formando grupos, Mulinum spinosum (Cav.) Pers., arbusto en cojín, Ephedra chilensis C.Presl Carex andina Phil., Euphorbia collina Phil., y Festuca acanthophylla E.Desv., en champas.

En la cima del cerro El Roble se realizan actividades de esparcimiento que podrían poner en peligro a la pequeña población, sin embargo el lugar ha sido declarado Santuario de la Naturaleza (Decreto Exento N²29, año 2000, del Ministerio de Educación) y el ingreso de visitantes está relativamente regulado. De todas formas C. espinosae es una especie Rara y debe ser considerada como emblemática dentro de un programa de monitoreo de la biodiversidad asociado a la condición de Santuario del lugar. En la zona de Pedernales hay una gran cantidad de ganado caprino que también podría poner en peligro dicha población.

\section{AGRADECIMIENTOS}

Se agradece a María Teresa Eyzaguirre por las fotos y material de Pedernales; a Sergio Elórtegui, Elizabeth Barrera, Víctor Ardiles y a Alejandra Vidal por las fotografías de la especie.

\section{BIBLIOGRAFIA}

Ehrhart, C. 2000. Die Gattung Calceolaria (Scrophulariaceae) in Chile. Bibliotheca Botanica, Heft 153 (I-VI), 1-283.

Espinosa, M. 1933 (publ. 1934). Sociedad Chilena de Historia Natural, Sesión ordinaria, en 15 de Noviembre de 1933. Revista Chilena de Historia Natural 37: 312-314. 